ENGLISCHE

LITER ATURGESCHICHTE 


\title{
ENGLISCHE LITERATURGESCHICHTE
}

\author{
unter Mitarbeit von \\ Stephan Kohl, Eberhard Kreutzer, Annegret Maack, \\ Manfred Pfister, Johann N. Schmidt und Hubert Zapf \\ herausgegeben von Hans Ulrich Seeber
}

2. Auflage 
Die einzelnen Kapitel bzw. Abschnitte wurden verfaßt:

Seite 1 bis 42 Stephan Kohl

Seite 43 bis 148 Manfred Pfister

Seite 149 bis 216 Johann N. Schmidt

Seite 217 bis 364 Hans Ulrich Seeber

Seite 364 bis 381 Hubert Zapf

Seite 381 bis 393 Annegret Maack

Seite 394 bis 438 Eberhard Kreutzer

Die Deutsche Bibliothek - CIP-Einheitsaufnahme

Englische Literaturgeschichte / unter Mitarb. von Stephan Kohl

... Hrsg. von Hans Ulrich Seeber. 2. Aufl. - Stuttgart ;

Weimar : Metzler, 1993

ISBN 978-3-476-00911-1

NE: Seeber, Hans Ulrich [Hrsg.]; Kohl, Stephan

ISBN 978-3-476-00911-1

ISBN 978-3-476-03475-5 (eBook)

DOI 10.1007/978-3-476-03475-5

Dieses Werk einschließlich aller seiner Teile ist urheberrechtlich geschützt. Jede Verwertung außerhalb der engen Grenzen des Urheberrechtsgesetzes ist ohne Zustimmung des Verlages unzulässig und strafbar.

Das gilt insbesondere für Vervielfältigungen, Übersetzungen, Mikroverfilmungen und die Einspeicherung und Verarbeitung in elektronischen Systemen.

(C) 1993 Springer-Verlag GmbH Deutschland Ursprünglich erschienen bei J. B. Metzlersche Verlagsbuchhundlung und Carl Ernst Poeschel Verlag GmbH in Stuttgart 1993 


\section{INHALTSVERZEICHNIS}

VORWORT IX

ALTENGLISCHE LITERATUR

(Stephan Kohl)

Historische Ausgangslage 1

Literarische Voraussetzungen 2

Der germanische Held 4

Christliche Gegenentwürfe 8

Heiligenleben 11

Bibeldichtung 13

Religiöse Dichtung 14

Prosa 16

\section{MITTELENGLISCHE LITERATUR}

(Stephan Kohl)

Historische Ausgangslage 19

Literarische Voraussetzungen 20

Frömmigkeit 23

Unterhaltung 27

Debatte und Gesellschaftsdichtung 36

DIE FRÜHE NEUZEIT: VON MORUS BIS MILTON

(Manfred Pfister)

Problematik der Periodisierung 43

Von der Reformation zur Revolution 46

Die Tudors: Mythos und Realpolitik 46 Zwei Nationen: Stuart und Commonwealth 51 Bestandsaufnahmen zur Lage der Nation $56 \mathrm{My}-$ then von Geschichte und Staat 59

Neue Orientierungen: Renaissance, Humanismus und Neue Wissenschaft 62 Humanistische Bildungsreformen 63 Utopische Entwürfe 68 Utopie und Neue Wissenschaft 71 Die Bilanzierung des Verlusts 74

Literatur als Institution und Medium 77

An der Schwelle zum literarischen Markt 77 Poetik: Von Normen und Formen 80

Realprosa und Weltaneignung 84

Die Vertextung der Neuen Welt 84 Der Essay und die politische Weltklugheit 89 
Das beredte Ich: Poetische Rollenspiele 92

Zeitgenössische Konstruktionen der Dichtungsgeschichte 93 Der Dialog mit Petrarca 94 Metaphysical Poetry 103

Erzählte Welt: Geschichten, Geschichte und Mythos 107

Auf dem Weg zum Roman? 107 Elisabeths England als Feenreich 109

Die Anatomie des Wit 111 Aufruhr in Arkadien 112 Satyr und Picaro in England 116 Das getaufte Epos 120

Inszenierte Wirklichkeit: Weltenbühne und Bühnenwelten 124

Ein Welttheater made in England 124 Weltgeschichte im "wooden O« 130 Tragische Fälle 134 Rachetragödien und tragische Rächer 137 Zwei Komödientraditionen 141 Tragical-comical-historicalpastoral 145

\section{VON DER RESTAURATION ZUR VORROMANTIK}

\section{(Johann N. Schmidt)}

Periodisierung 149

Entstehung von »literarischer Öffentlichkeit « 155

Newton, die "Neue Naturwissenschaft " und das philosophische Zeitalter 161

Versdichtung 165

Imitation und Parodie 165 Pastoraldichtung und Lehrgedicht 168 Vom Klassizismus zur Vorromantik 172 Robert Burns 174

Romanentwicklung 175

Daniel Defoe: Moralisches Exempel und die Abenteuergeschichte 179 Richardson und der Briefroman 182 Fieldings "comic epic poem in prose 186 Sterne und der "sentimentale Roman" 189 Romanentwicklung 1750-1800 191

Satire 195

Satire von Butler bis Pope 198 Swifts Prosasatiren 200 Bild- und Verssatire nach Swift 203

Moralische Wochenschriften 204

Das Restaurationsdrama 207

Das heroische Drama 207 Die Restaurationskomödie 209

Die Sentimentalisierung von Komödie und Tragödie 213

\section{ROMANTIK UND VIKTORIANISCHE ZEIT}

(Hans Ulrich Seeber)

Modernisierung und Literatur im 19. Jahrhundert 217

Die Literatur der Romantik 223

Die Besonderheit der englischen Romantik 223 Wege zur Romantik 225 Antworten auf die Modernisierung. Poetischer Individualismus, Natur als Ersatzreligion, Imagination 227 Wordsworth und Coleridge 235 Keats, Shelley, Byron, Clare 242 Prosa 253

Der Roman des 19. Jahrhunderts 255

Roman und Gesellschaft 255 Der Schauerroman 262 Der historische Roman 265 Der Sozialroman 269 Charles Dickens und London 272 Realismus bei Thackeray, Trollope und Meredith 275 Romane von Frauen. Jane Austen, die Brontës, George Eliot 279

Fortschrittsglaube und Kulturkritik. Zur Prosa der Viktorianer 285 
Die Lyrik der Viktorianer 292

Überblick 292 Melancholie und Energie. Frühviktorianische Versdichtung 293 Die Präraphaeliten 298 Englishness als Weg ins 20. Jahrhundert 302

\section{VORMODERNE UND MODERNE}

\section{(Hans Ulrich Seeber)}

Die Krise des Liberalismus und der Modernisierung 306

Die Literatur der Übergangszeit um 1900309

Literatur und Imperialismus 310 Ästhetizismus und Naturalismus in den >Nineties 313 Pessimismus, Perspektivismus und der Beginn der modernen Romankunst (Hardy, Conrad, James) 316 Evolution, Utopie und Literatur 319

Keltische Renaissance und irische Literatur 322

Shaw und die Erneuerung des britischen Dramas 326

Die modernistische Revolution um 1910331

Reaktionen auf den Ästhetizismus oder der Schein des Lebens 331 Gebremste Modernität. Georgian Poetry, Kriegsdichtung, Imagismus 332 Ästhetischer Modernismus und Kulturkritik (Lyrik und Literaturkritik) 335 Der experimentelle Roman 340 Tradition und Moderne im Roman 345

Wirtschaftskrise, politische Radikalisierung und Literatur in den dreißiger Jahren 349

\section{DIE ZEIT NACH 1945}

\section{(Hans Ulrich Seeber/Hubert Zapf/Annegret Maack)}

Großbritannien auf der Suche nach einer neuen Rolle 352

Die Lyrik nach 1945355

Überblick 355 Das Movement der fünfziger Jahre 357 Reaktionen auf das Movement 359

Das Drama nach 1945364

Das englische Drama bis 1956364 Die Angry Young Men und die Geburt des modernen englischen Dramas 365 Außeneinflüsse: Amerikanisches Drama, Episches Theater 366 Samuel Beckett und das absurde Theater 367 Das Neue Englische Drama und die Problemstruktur einer sabstrakten Gesellschaft 369 Harold Pinter: Dramatischer Hyperrealismus 370 Tom Stoppard und Edward Bond. Spielästhetisches vs. politisches Drama 372 Die Second Wave des englischen Dramas seit 1968376 Das englische Drama in den 80er Jahren 379

Der Roman nach 1945381

Nach dem Krieg: Die 50er Jahre 381 Nach den 50er Jahren: Ethik und Ästhetik im Roman von Golding, Lessing, Murdoch, Spark 384 Auf dem Weg zur Postmoderne 390 


\section{COMMONWEALTH-LITERATUR}

\section{(Eberhard Kreutzer)}

Die weltweite Auffächerung englischsprachiger Literatur in nachkolonialer Zeit 394

Terminologische Abgrenzungen und Zuordnungsprobleme 396

Kulturelle Determinanten und historischer Hintergrund 400

Literarhistorische Entwicklungstendenzen und Bedingungen des Literaturbetriebs 403

Aspekte der Sprachenwahl und varietätenspezifischen Stilisierung 407

Die Wiederbelebung autochthoner Traditionen in den Konzepten einer neuen orature 411

Die Revision des Kanons englischer Klassiker 414

Die literarische "Kartographie « unerschlossener Territorien 416

Literarischer Regionalismus in Kanada 419

Insularität und Wanderwege in der karibischen Literatur 421

Die Aufarbeitung der Kolonialgeschichte in der afrikanischen Literatur 423

Die Bloßstellung nachkolonialer Fehlentwicklungen in der Literatur der Dritten Welt 425

Literatur im Zeichen der Apartheid 429

Antipoden-Konzepte in der Literatur Australiens und Neuseelands 431

Angestammte und moderne Welt am Beispiel der Ost-West-Begegnung in der indischen Literatur 433

Die Situation der Frau und Ansätze einer Frauenliteratur 435

Die Tendenz des "Multikulturalismus «: das Aufkommen ethnischer Minoritätenliteraturen 437

BIBLIOGRAPHIE 439

REGISTER 447

BILDQUELLEN 461 


\section{VORWORT}

Ein Granitblock, der unterschiedlichen Betrachtern immer das gleiche Bild darböte, ist die historische Vergangenheit nicht. Eher gleicht sie dem Spiel des Wassers, dem wechselnde Lichtverhältnisse immer neue Farbtöne entlokken. Nicht nur fördert die Wissenschaft ständig neue Tatsachen und Einsichten zutage, auch die Seh- und Interpretationsweise verschiedener Generationen von Lesern ist erheblichen Wandlungen unterworfen. Die Einsicht in das dynamische Wechselverhältnis von Gegenwart und Vergangenheit stand am Anfang dieser Literaturgeschichte, die von der Überzeugung ausgeht, daß Geschichte immer wieder neu erarbeitet, erzählt, d.h. konstruiert werden muß. Was beispielsweise zum Kanon des Nachwirkenden, Gelungenen und Relevanten gehört, steht nicht ein für allemal fest. Die Autoren dieses Bandes nahmen sich die Freiheit, bei der Auswahl und Gewichtung von Gattungen, Autoren und Werken hier und dort eigene Akzente zu setzen, hielten es aber nicht für ratsam, die in einer langen Wirkungsgeschichte entstandene Übereinkunft über bedeutende Vertreter und Texte der englischen Literatur zu übersehen. Es war ihre Absicht, den eindrucksvollen Reichtum und die Vielfalt einer Literatur sichtbar zu machen, die schon seit dem Mittelalter (Chaucer), der frühen Neuzeit (Shakespeare) und dem 18. Jahrhundert (Defoe) die Vorstellung der englischen und nicht-englischen Leser von dem, was etwa Drama und Erzählung zu leisten vermögen, entscheidend geprägt hat.

Indem Literatur den gesellschaftlichen und kulturellen Wandel abbildend, deutend und kommentierend begleitet, gestaltet sie ihn auch mit. Eine allgemein verbindliche Theorie dieses Wandels gibt es freilich nicht. Man könnte sogar mit Thackeray die Auffassung vertreten, bei der Geschichte handele es sich lediglich um ein Theater, das mit wechselnden Besetzungen und Kostümen immer wieder dasselbe traurige Stück von Aufschwung und Fall, von utopischer Überschwenglichkeit und endlicher, vom Zufall bestimmter Wirklichkeit zur Aufführung bringt. Als Hypothese für eine Literaturgeschichte wäre eine solche Annahme aber wenig hilfreich. Ein Leitmotiv des Zusammenhangs, den die vorliegende Darstellung erzählend und argumentierend zu stiften versucht, ist dagegen die Modernisierungstheorie. Es wird also zum Beispiel danach gefragt, was es für die englische Literatur bedeutet, daß sie vor allem seit der Renaissance im Zeichen rascher Modernisierungsschübe - Rationalisierung, Säkularisierung, Nationalstaatsbildung, industrielle Revolution, Demokratisierung, funktionale Ausdifferenzierung von Gesellschaft und Kultur, Individualisierung - steht. Dabei erlaubt der Bezug auf den Begriff der Modernisierung eine Auflockerung der starren Gegenüberstellung Sozialgeschichte vs. Geistesgeschichte. Am komplexen Spiel der Wechselwirkungen und Diskurse, in dem sich geschichtliche Wirklichkeit konstituiert, sind beide Dimensionen entscheidend beteiligt.

Diese Anbindung an den "außerliterarischen« Kontext der Umwandlung einer von Traditionen geprägten Gesellschaft in eine moderne will nun nicht 
besagen, daß sich für die Beiträger zu diesem Buch Literatur darin erschöpft, Zeugnis für etwas zu sein, was längst vergangen ist oder in vielfach vermittelter und verwandelter Form weiterlebt. Die Art und Weise, wie Kunstwerke Erfahrungen und Wertvorstellungen ihrer jeweiligen Zeit gestalten, ermöglicht es späteren Lesern in besonderem Maße, diese auf eigene Erfahrungen und Bedürfnisse zu beziehen.

Der Titel »englische Literaturgeschichte« setzt eine Konvention der Literaturgeschichtsschreibung fort, für die es keine überzeugende Alternative gibt. Es muß aber ausdrücklich gesagt werden, daß die "englische « Literatur ohne ihre zahlreichen Autoren irischer, schottischer, walisischer, amerikanischer, westindischer, indischer, afrikanischer etc. Herkunft sehr viel ärmer wäre. Tatsächlich sind seit geraumer Zeit Literatur und Kultur jene Bereiche, in denen sich der Selbständigkeitsanspruch etwa der keltischen Randgebiete besonders deutlich äußert. Die Wichtigkeit der englischsprachigen Commonwealth-Literatur und deren vielfältige Wechselwirkungen mit der literarischen Kultur der Insel sind so auffällig, daß ihr das abschließende Kapitel dieses Buches gewidmet wird.

Der begrenzte Umfang dieses Buches ließ keine "flächendeckende" Darstellung zu, die mit zureichender Ausführlichkeit auf alle jene Werke und Autoren einginge, welche man in einer Literaturgeschichte herkömmlicher Art vielleicht erwarten würde. Die Absicht der Verfasser war es vielmehr, typische Entwicklungen, Gattungen, Wissensbereiche und Autoren beispielhaft herauszuarbeiten und das funktionale Verhältnis von Text und Kontext sichtbar zu machen. Dabei kam es nicht so sehr darauf an, für jede Epoche ein möglichst einheitliches Bild zu entwerfen, sondern gerade auch Konflikte und gegenläufige Strömungen aufzuzeigen. Der Zwang zur Beschränkung und die Erfahrung, daß viele frühe englische Texte nicht in gleicher Weise in einem lebendigen, auf die Gegenwart bezogenen Traditionszusammenhang stehen wie etwa die Werke von Shakespeare oder Morus, führte dazu, den Anteil der Literatur vor 1500 drastisch zu kürzen.

Der methodische Ansatz dieses Buches und seine an der Gliederung ablesbaren Einteilungen des zeitlichen Ablaufs wurden von den Beiträgern bewußt gewählt. Diese Literaturgeschichte kann und will nicht jene gedankliche und stilistische Einheitlichkeit bieten, die nur ein einzelner Autor garantieren kann. Dies ist keineswegs nur ein Nachteil. Im Spiegel individueller Temperamente kommen der Reichtum und die Vielfalt der englischen Literatur eher besser zur Geltung.

Der Herausgeber dankt all jenen, die das Zustandekommen dieses Bandes ermöglichten. Bernd Lutz - von dem die Anregung kam - und Petra Wägenbaur vom Metzler Verlag haben das Entstehen dieses Buches mit Sympathie, Geduld und großer Hilfsbereitschaft begleitet. Sehr dankbar bin ich den KoAutoren, die sich teilweise kurzfristig zur Mitarbeit entschlossen und mir meine Arbeit leicht machten. Ihre Loyalität und ihre Bereitschaft, neue Wege zu gehen, waren eine große Hilfe. Edmund Stegmaier danke ich für freundschaftlichen Rat und steten Zuspruch. Großes Lob haben schließlich Elisabeth Hartel, Dietmar Jaegle und vor allem Monika Lacher verdient, die unermüdlich halfen, einen druckreifen Text zu erstellen. 